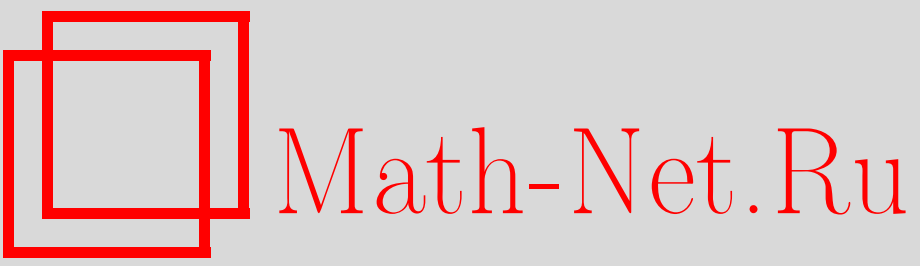

И. Н. Никитин, Частные классы движений струны, квантуемые без аномалий, TMФ, 1996, том 109, номер 2, 202-214

DOI: https://doi.org/10.4213/tmf1222

Использование Общероссийского математического портала Math-Net.Ru подразумевает, что вы прочитали и согласны с пользовательским соглашением

http://www.mathnet.ru/rus/agreement

Параметры загрузки:

IP : 54.157 .27 .8

26 апреля 2023 г., $12: 56: 47$ 
ТЕОРЕТИЧЕСКАЯ

И МАТЕМАТИЧЕСКАЯ

ФИЗИКА

Том 109, № 2

ноябрь, 1996

И.Н. Никитин ${ }^{*}$

\section{ЧАСТНЫЕ КЛАССЫ ДВИЖЕНИЙ СТРУНЫ, КВАНТУЕМЫЕ БЕЗ АНОМАЛИЙ}

Исследованы конечномерные подмногообразия фазового пространства открытой бозонной струны, квантование которых свободно от аномалий при $d=4$.

\section{ВВЕДЕНИЕ}

В работах $[1,2]$ отмечалась принципиальная возможность построения квантовой теории струн без аномалий в пространстве Минковского произвольной размерности, в частности в 4 измерениях. Для построения этой теории предлагается использовать следующие ключевые идеи.

1. Воспользуемся нековариантным гамильтоновым формализмом: репараметризационную симметрию теории зафиксируем наложением калибровок, на мировых листах струн введем определенную параметризацию.

2. Эта параметризация должна быть лоренц-инвариантной. В теории струн обычно используется лоренц-неинвариантная параметризация мирового листа "световыми" координатами, называемая калибровкой светового конуса. В этом подходе преобразования Лоренца изменяют положение мирового листа относительно неподвижной оси калибровки, они сопровождаются репараметризациями, вследствие чего квантовая аномалия в группе репараметризаций приводит к аномалии в группе Лоренца и потере лоренц-ковариантности теории. Лоренц-инвариантную параметризацию мирового листа нетрудно построить, связав ось калибровки с сохраняющимся вектором в теории струны, например с полным импульсом (калибровка Рорлиха в [1]) или спином ("динамическая калибровка светового конуса" в [2]). В этом случае преобразования Лоренца одновременно воздействуют на мировой лист и ось калибровки, при этом параметризация мирового листа сохраняется.

3. Для того чтобы получить явно лоренц-ковариантную теорию, при квантовании следует использовать набор независимых канонических переменных, явно содержащий генераторы преобразований Лоренца. В этом случае коммутационные соотношения для генераторов будут постулированы непосредственно из скобок Пуассона, и аномалий не

\footnotetext{
${ }^{*}$ Институт физики высоких энергий
} 
возникнет. Как показывает более детальное исследование, достаточно, чтобы независимыми переменными были генераторы врашений в системе покоя (спин струны). Остальные независимые переменные должны обладать простыми скобками Пуассона. Такой набор переменных построен в [1], он состоит из конечного набора, содержашего генераторы врашений и трансляций, канонически сопряженные к ним переменные, определяющие ориентацию и общее положение струны, и независимого от него бесконечного набора лоренц-инвариантных переменных “действие-угол”. Единственная трудность, препятствуюшая квантованию теории в этом подходе, состоит в том, что переменные действие-угол имеют сложные области изменения, точная форма которых не изучена. Поскольку свойства квантовой теории сушественно зависят от глобальной топологической структуры фазового пространства, эту структуру необходимо исследовать, а также использовать оставшийся произвол в выборе независимых канонических переменных так, чтобы сделать ее максимально простой.

В настояшее время данная программа не реализована полностью. Квантовая теория построена только для подмногообразий фазового пространства, представляюших определенные частные классы движения струн. Наиболее полно изучено 4-мерное многообразие, на котором струна имеет вид прямолинейного стержня, врашаюшегося с постоянной угловой скоростью в системе покоя. Квантовая теория прямолинейной струны [3] обладает спин-массовым спектром, состояшим из одной ведушей реджевской траектории. В работе [2] проведено квантование бесконечномерных многообразий, которым отвечают мировые листы, обладающие осевой симметрией. Спин-массовый спектр этой теории содержит бесконечный набор реджевских траекторий.

В данной работе исследуется класс конечномерных многообразий в фазовом пространстве струны, которые в обшем случае не являются подмножествами осесимметричных и каждое из которых содержит прямолинейные струны. В работе используется гамильтонова механика струны в калибровке Рорлиха [1], описанная в разделе 1. В разделе 2 в фазовом пространстве струны выделены 6-мерные многообразия (ограниченные конфигурации) и показано, что эти многообразия локально изоморфны фазовому пространству системы “1-мерный осциллятор +3 -мерный ротатор". В раздле 3 показано, что при определенных ограничениях топологического характера эти многообразия глобально изоморфны фазовому пространству системы “осциллятор+ротатор". В разделе 4 проведено квантование этих многообразий, получены спин-массовые спектры и исследованы дискретные симметрии системы.

\section{1. ФАЗОВОЕ ПРОСТРАНСТВО СТРУНЫ}

Фазовым пространством открытой струны является множество $\mathcal{M}$ всевозможных замкнутых ориентированных кривых $\vec{Q}(\sigma)$ с отмеченной точкой $\vec{Q}(0)$. Эти кривые в дальнейшем именуются опорными. Параметрические инварианты опорных кривых являются сохраняюшимися динамическими переменными в теории струны, например, длина кривой равна удвоенной массе струны $L=2 M$, ориентированная плошадь поверхности, натянутой на кривую, не зависит от поверхности и равна удвоенному спину струны $\vec{A}=2 \vec{S}$. На опорных кривых введена натуральная параметризация: $\sigma=2 \pi l / L$, $l$ - длина дуги кривой, отсчитываемая от отмеченной точки; $\left|\vec{Q}^{\prime}\right|=M / \pi$. Опорные 
кривые являются траекториями концов струны в системе покоя, причем $\vec{Q}(0)$ является положением конца в текущий момент времени. Форма струны определяется формулой (см. рис. 1)

$$
\vec{x}(\sigma)=(\vec{Q}(\sigma)+\vec{Q}(-\sigma)) / 2, \quad 0 \leq \sigma \leq \pi
$$

эволюция сводится к равномерному движению отмеченной точки по неподвижной опорной кривой: $\vec{Q}(\sigma) \rightarrow \vec{Q}(\sigma+\tau)$.

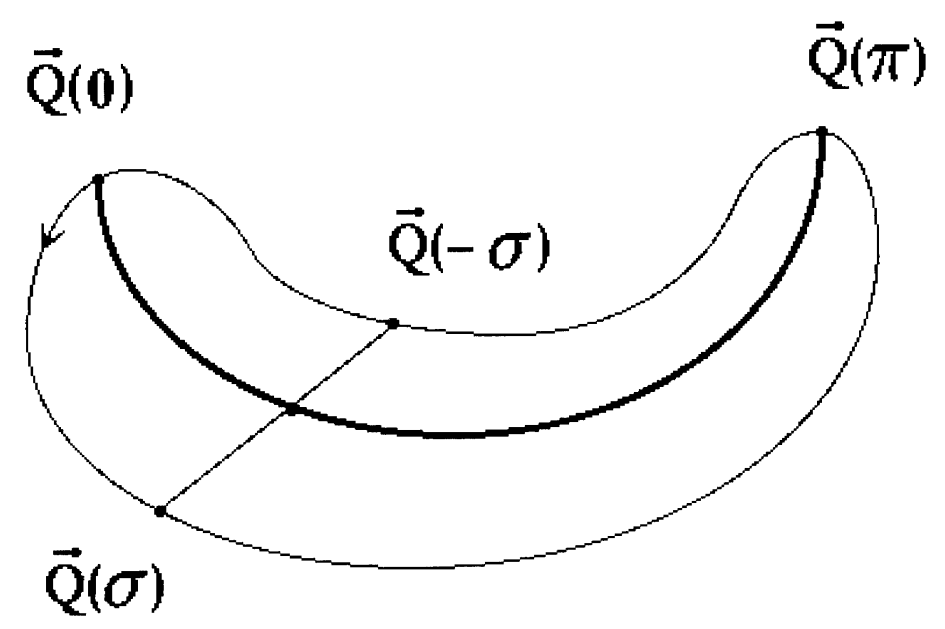

Рис. 1. орма струны определяется следующим образом: отложим на опорной кривой две дуги равной длины, лежащие по разные стороны от точки $\vec{Q}(0)$; соединим концы дуг прямолинейным отрезком и отметим его середину. Множество таких середин описывает струну в момент времени, когда ее конец находится в точке $\vec{Q}(0)$.

Симплектическая структура фазового пространства определяется замкнутой 2-формой

$$
\omega=-\frac{1}{4} \int_{0}^{2 \pi} d \sigma \delta \vec{Q}^{\prime}(\sigma) \wedge \delta \vec{Q}(\sigma)
$$

Факторизуем $\mathcal{M}$ по действию группы $\Gamma=S O(3) \times \mathbf{R}^{3} \times S^{1}$ врашений, трансляций и сдвигов отмеченной точки. Произвольную опорную кривую представим в виде

$$
\vec{Q}(\sigma)=\vec{e}_{i} Q_{i}(\sigma+\tau ; u)+\vec{D},
$$

ортонормированный репер $\vec{e}_{i}$ и вектор $\vec{D}$ задают положение опорной кривой, $\tau$ определяет сдвиги отмеченной точки. Переменные $u \in \mathcal{M} / \Gamma$ определяют форму опорной кривой - среди всех кривых заданной формы, отличаюшихся на врашения, транслящии 
и сдвиги отмеченной точки, выбран один представитель $Q_{i}(\sigma)$ (этот выбор будет конкретизирован ниже). Подставляя (2) в форму (1), получим

$$
\begin{gathered}
\omega=\omega_{M}+\omega_{S}+\omega_{u} \\
\omega_{M}=d \tau \wedge d \mu, \quad \omega_{S}=\frac{1}{2} d \vec{e}_{i} \wedge d\left(\vec{S} \times \vec{e}_{i}\right) \\
\omega_{u}=-\frac{1}{4} \int_{0}^{2 \pi} d \sigma \delta Q_{i}^{\prime}(\sigma) \wedge \delta Q_{i}(\sigma), \quad \mu=\frac{M^{2}}{2 \pi} \\
\vec{S}=-\frac{1}{4} \int_{0}^{2 \pi} d \sigma \vec{Q}^{\prime} \times \vec{Q}
\end{gathered}
$$

- спин струны.

Переменная $\vec{D}$ выпадает из формы и не участвует в механике. Положим $\vec{D}=0$ (положение $Q_{i}(\sigma)$ зафиксируем условием $\left.\int_{0}^{2 \pi} d \sigma Q_{i}=0\right)$.

Форма $\omega_{M}$ определяет гамильтонову механику осциллятора. В подходе [1] гамильтониан струны $H=\mu$, отсюда видно, что эволюция сводится к сдвигу $\tau \rightarrow \tau+\Delta \tau$.

Форма $\omega_{S}$ определяет механику волчка. Направим $\vec{e}_{3}$ вдоль $\vec{S}: \vec{e}_{3}=\vec{S} / S, \vec{e}_{1}=\vec{e}$, $\vec{e}_{2}=\vec{e}_{3} \times \vec{e}_{1}$ (ориентацию $Q_{i}(\sigma)$ зафиксируем условием $\left.\int_{0}^{2 \pi} d \sigma Q_{3}^{\prime} Q_{i}=0, i=1,2\right) . \mathrm{B}$ этом случае $\omega_{S}$ превращается в симплектическую форму ротатора:

$$
\omega_{S}=d \vec{e} \wedge d(\vec{S} \times \vec{e}), \quad \vec{e}^{2}=1, \quad \vec{S} \vec{e}=0 .
$$

Форма $\omega_{u}$ определяет симплектическую структуру фактор-пространства $\mathcal{M} / \Gamma$. Формы $\omega_{M}, \omega_{S}$ и $\omega_{u}$ не являются независимыми, поскольку в набор $u$ входят переменные $\mu$ и $S$. Добавление $\omega_{u} \mathrm{k} \omega_{M}+\omega_{S}$ меняет некоторые скобки Пуассона в механике $\omega_{M}+\omega_{S}$ (в частности, $\{\tau, \vec{e}\})$. Форма $\omega_{u}$ не содержит переменных $\tau$ и $\vec{e}_{i}$, она сохраняется при преобразованиях, генерируемых $\mu$ и $\vec{S}$ в механике $\omega_{M}+\omega_{S}$ (при сдвигах $\tau$ и врашениях $\vec{e}_{i}$ ). Поэтому в механике $\omega_{M}+\omega_{S}+\omega_{u}$ переменные $\mu$ и $\vec{S}$ генерируют те же преобразования:

$$
\{\mu, \tau\}=1, \quad\left\{S^{i}, S^{j}\right\}=-\epsilon_{i j k} S^{k}, \quad\left\{S^{i}, e^{j}\right\}=-\epsilon_{i j k} e^{k},
$$

остальные скобки Пуассона $\{\mu, \ldots\},\{\vec{S}, \ldots\}$ равны нулю.

Канонические преобразования фазового пространства $\delta u=\alpha X$ с гамильтонианом $H: i_{X} \omega=d H$ сохраняют симплектическую форму: $\delta \omega=0$. Преобразования с динамическим параметром $\delta u=\alpha(u) X$ меняют $\omega$ на полный дифференциал: $\delta \omega=\mathcal{L}_{\alpha X} \omega=d\left(\alpha i_{X} \omega\right)=d \alpha \wedge d H$ (использованы соотношения $\mathcal{L}_{X}=i_{X} d+d i_{X}$ и $d \omega=0)$. При добавлении к $\omega$ слагаемого $\Delta \omega$, сохраняющегося при преобразованиях $\delta u=\alpha(u) X: \forall \alpha(u) \delta \Delta \omega=0$, генератор $H$ этих преобразований не меняется.

Восстановление механики до 4-мерной проводится следующим образом. Вводятся канонические переменные $Z_{\mu}, P_{\mu}$ - средняя координата и полный 4-импульс струны, в симплектическую форму добавляется слагаемое $d P_{\mu} \wedge d Z_{\mu}$. Опорная кривая в пространстве Минковского определяется выражением

$$
Q_{\mu}(\sigma)=X_{\mu}+P_{\mu}\left(\frac{\sigma}{\pi}-1\right)-\vec{N}_{\mu} \vec{Q}(\sigma)
$$


где $N_{\mu}^{i}=N_{\mu}^{i}(P): N_{\mu}^{i} P_{\mu}=0, N_{\mu}^{i} N_{\mu}^{j}=-\delta^{i j} ; \quad X_{\mu}=Z_{\mu}-\frac{1}{2} \epsilon_{i j k} \Gamma_{\mu}^{i j} S^{k}, \quad \Gamma_{\mu}^{i j}=$ $N_{\nu}^{i} \partial N_{\nu}^{j} / \partial P_{\mu}$. Опорная кривая является мировой линией конца струны. Она светоподобна: $Q^{\prime 2}=0$. Мировой лист струны определяется выражением $x_{\mu}\left(\sigma_{1}, \sigma_{2}\right)=\left(Q_{\mu}\left(\sigma_{1}\right)+\right.$ $\left.Q_{\mu}\left(\sigma_{2}\right)\right) / 2$ (геометрическое место середин отрезков, соединяющих всевозможные пары точек на опорной кривой), сечение мирового листа плоскостью $x_{0}=$ const дает струну в этот момент времени. Гамильтонианом системы является связь $H=\left(P^{2}-M^{2}\right) / 2 \pi \approx 0$, генерируюшая сдвиги $Q_{\mu}(\sigma) \rightarrow Q_{\mu}(\sigma+\tau)$.

Генераторы группы Лоренца определяются выражением

$$
M_{\mu \nu}=X_{\mu} P_{\nu}-X_{\nu} P_{\mu}+\epsilon_{i j k} N_{\mu}^{i} N_{\nu}^{j} S^{k}
$$

Эти переменные генерируют преобразования Лоренца вектора $X_{\mu}$ и системы координат $\left(P_{\mu} / \sqrt{P^{2}}, \vec{N}_{\mu} \vec{e}_{i}\right)$. Вектор $Q_{\mu}(\sigma)$ разложен по этой системе координат с коэффициентами $\left(M\left(\frac{\sigma}{\pi}-1\right), Q_{i}(\sigma+\tau)\right)$, сохраняюшимися при преобразованиях Лоренца. В нашем подходе $Z_{\mu}, P_{\mu}$ и $\vec{S}$ являются независимыми каноническими переменными, квантовые коммутационные соотношения для которых постулируются из скобок Пуассона. Нетрудно проверить, что в квантовой механике алгебра Лоренца $M_{\mu \nu}$ замкнута и $Q_{\mu}$ преобразуется как 4-вектор.

Переменные $u$ параметризуют симплектическое многообразие $\mathcal{M} / \Gamma$. В работах [1] на картах этого многообразия введены симплектические координаты и исследована структура полноразмерной окрестности прямолинейной струны. Глобальная структура этого многообразия не изучена. Мы будем рассматривать в $\mathcal{M}$ подмногообразия вида $u=u(\mu, S)$.

\section{2. ОГРАНИЧЕННЫЕ КОНФИГУРАЦИИ}

Рассмотрим семейство $\mathcal{M}_{2}$ опорных кривых, форма которых однозначно определяется двумя параметрами - массой и спином струны (длиной и плошадью опорной кривой). Примером является семейство эллипсов. В $\mathcal{M}_{2}$ включаются опорные кривые заданной формы со всевозможными значениями $\tau$ и $\vec{e}_{i}$. Поэтому выделенные подмногообразия фазового пространства сохраняются при эволюции и при поворотах. Фактически мы рассматриваем теорию частного класса движений струны, в которой имеется врашательная инвариантность.

С учетом переменных, определяюших ориентацию и выбор отмеченной точки, такое семейство обладает шестью степенями свободы. Подстановка выражения $Q_{i}=$ $Q_{i}(\sigma ; \mu, S)$ в третье слагаемое формы (3) приводит это слагаемое к виду $g(\mu, S) d S \wedge d \mu$, где

$$
g(\mu, S)=\frac{1}{2} \int_{0}^{2 \pi} d \sigma \frac{\partial Q_{i}^{\prime}}{\partial \mu} \frac{\partial Q_{i}}{\partial S} .
$$

$\omega_{u}$ можно записать в виде $d F \wedge d \mu+d G \wedge d S$, где

$$
F=-\frac{1}{4} \int_{0}^{2 \pi} d \sigma Q_{i}^{\prime} \frac{\partial Q_{i}}{\partial \mu}+\frac{\partial K}{\partial \mu}, \quad G=-\frac{1}{4} \int_{0}^{2 \pi} d \sigma Q_{i}^{\prime} \frac{\partial Q_{i}}{\partial S}+\frac{\partial K}{\partial S} .
$$


Эти два слагаемых можно включить в $\omega_{M}$ и $\omega_{S}$, при этом в формах переопределятся $\tau$ и $\vec{e}:{ }^{1)}$

$$
\tau^{*}=\tau+F, \quad \vec{e}^{*}=R(G) \vec{e}
$$

Таким образом, на рассматриваемых подмногообразиях фазового пространства симплектическая форма имеет вид "осциллятор + ротатор":

$$
\omega=d \tau^{*} \wedge d \mu+d \vec{e}^{*} \wedge d\left(\vec{S} \times \vec{e}^{*}\right) .
$$

Этот результат не зависит от выбора семейства опорных кривых.

Произвольная функция $K$ в (5) определяет канонические преобразования переменных $\tau^{*}$ и $\vec{e}^{*}$. При преобразовании

$$
\tau \rightarrow \tau+\delta \tau, \quad \vec{e} \rightarrow R(\delta \alpha) \vec{e}, \quad Q_{i}(\sigma) \rightarrow R_{i j}(-\delta \alpha) Q_{j}(\sigma-\delta \tau)
$$

$(\delta \tau, \delta \alpha$ зависят от $\mu, S)$ меняется представитель $Q_{i}(\sigma)$ в классе эквивалентности $\mathcal{M}_{2} / \Gamma$ и не меняется $\vec{Q}(\sigma) \in \mathcal{M}_{2}$. Нетрудно убедиться, что изменение переменных $\left(\tau^{*}, \vec{e}^{*}\right)$ при этом преобразовании можно скомпенсировать каноническим преобразованием с $K=-\mu \delta \tau-S \delta \alpha$. Поэтому канонические переменные $\left(\tau^{*}, \vec{e}^{*}\right)$ не зависят от выбора представителя $Q_{i}(\sigma)$, вектор $\vec{e}^{*}$ и точка $\left.\vec{Q}(0)\right|_{\tau^{*}=0}$ связаны с кривой $\vec{Q}(\sigma)$ инвариантным образом. Мы выберем $\vec{e}=\vec{e}^{*}, \tau=\tau^{*}$ и не будем делать различия между этими переменными.

\section{3. ГЛОБАЛЬНАЯ СТРУКТУРА ФАЗОВОГО ПРОСТРАНСТВА}

В теории струн масса и спин удовлетворяют неравенству $\mu \geq S$ (условие Редже). Геометрически это условие означает, что среди всех замкнутых кривых заданной длины максимальная ориентированная плошадь достигается на окружности. Таким образом, $\mathcal{M}_{2}$ занимает область в прямом произведении фазовых пространств осциллятора и ротатора. Имеется еше одно важное отличие $\mathcal{M}_{2}$ от

$$
\mathbf{R}^{2}(\mu, \tau) \times T M\left(S^{2}\right)(\vec{e}, \vec{S})
$$

Пусть опорная кривая является ( $k$ раз сложенной) окружностью. В этом случае струна является прямолинейной ( $k$ раз сложенной). Преобразование $\tau \rightarrow \tau+C$, $\vec{e} \rightarrow R(-k C) \vec{e}$ сохраняет такие опорные кривые (см. рис. 2). Для прямолинейных струн точки фазового пространства, связанные этим преобразованием, необходимо отождествить. Прямолинейные струны выполняют роль склеек в фазовом пространстве. Если семейство опорных кривых содержит окружности, то $\mathcal{M}_{2}$ обладает топологической структурой, отличной от (7).

Далее мы рассмотрим многообразия $\mathcal{M}_{2}$, которые можно взаимно однозначно канонически отобразить в фазовое пространство системы "осциллятор + ротатор" (без ограничений на энергию осциллятора и спин ротатора).

\footnotetext{
${ }^{1)}$ В данной работе $R(\alpha)$ обозначает вращение вокруг направления $\vec{S}$ на угол $\alpha$.
} 

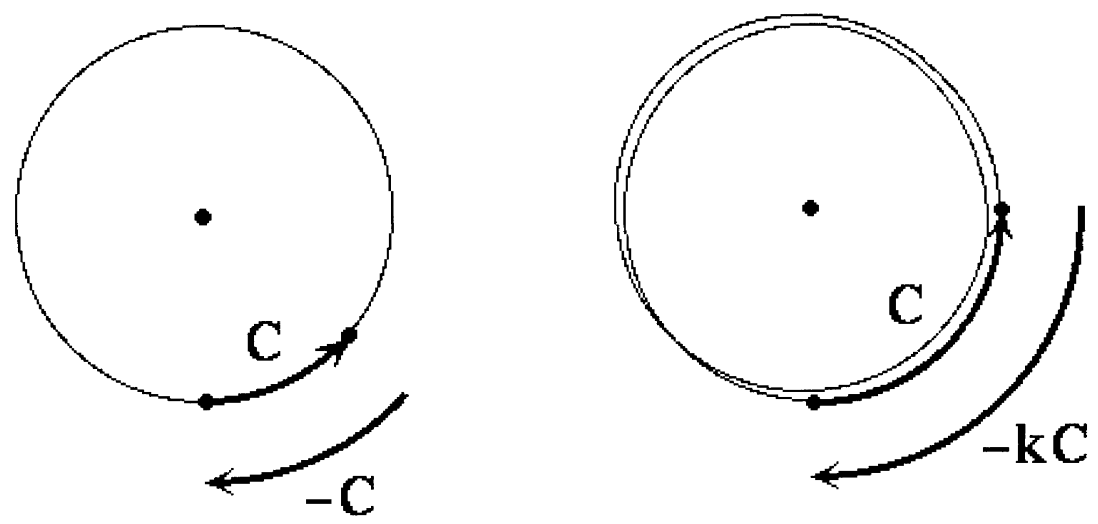

Рис. 2. Эволюция и поворот сохраняют окружности.

Рассмотрим семейство опорных кривых, для которого область изменения переменных $(\mu, S)$ имеет вид $\mu \geq k S$ (рис. 3 ). Отображение в фазовое пространство "осциллятор+ротатор" $\mathcal{F}$ имеет вид

$$
(\mu, \tau, \vec{S}, \vec{e}) \rightarrow(I, \tau, \vec{S}, \vec{n}),
$$

$\left(I \geq 0, \tau \in S^{1}\right)$ - переменные действие-угол для осциллятора,

$$
\mu=k S+I, \quad \vec{n}=R(k \tau) \vec{e} .
$$

Отображение взаимно однозначно, только если $k \in \mathbf{N}$. При этом на верхней границе области струна должна быть прямолинейной ( $k$ раз сложенной).

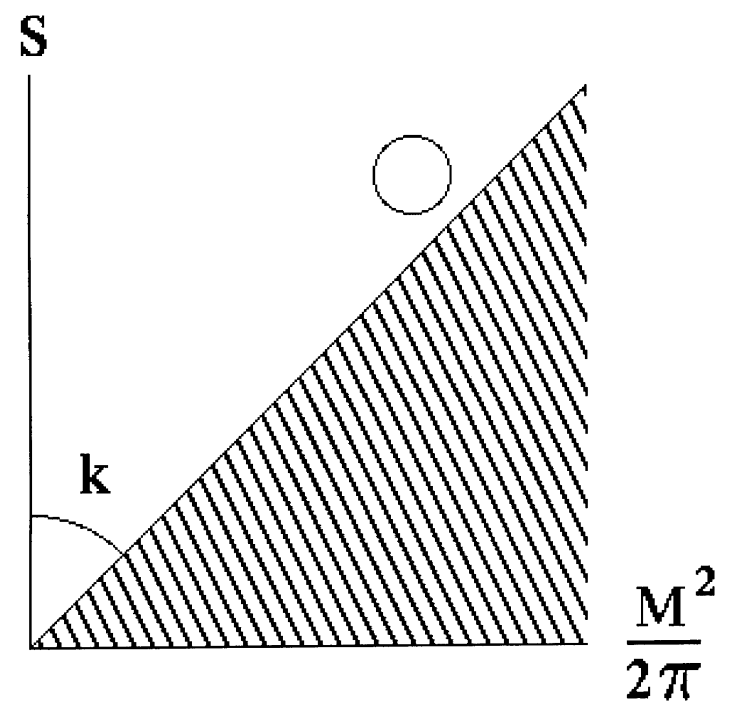

Рис. 3. Область Редже для конфигураций типа "осциллятор+ротатор". 
Положим $\mu=f(I, S)$. Подставляя это выражение в форму (6), получим

$$
\omega=d \tilde{\tau} \wedge d I+d \vec{n} \wedge d(\vec{S} \times \vec{n}), \quad \tilde{\tau}=f_{I}^{\prime} \tau, \quad \vec{n}=R\left(f_{S}^{\prime} \tau\right) \vec{e}
$$

Форма определяет скобки Пуассона 2 :

$$
\{I, \tilde{\tau}\}=1, \quad\left\{S^{i}, S^{j}\right\}=-\epsilon_{i j k} S^{k}, \quad\left\{S^{i}, n^{j}\right\}=-\epsilon_{i j k} n^{k} .
$$

Изменение $\tau$ на $2 \pi$ не меняет конфигурацию. Это преобразование должно быть тождественным в $\mathcal{F}$. Отсюда следует, что $f_{I}^{\prime}, f_{S}^{\prime} \in \mathbf{Z}$. Обратное отображение $\tau=\tilde{\tau} / f_{I}^{\prime}$, $\vec{e}=R\left(-f_{S}^{\prime} / f_{I}^{\prime} \cdot \tilde{\tau}\right) \vec{n}$ однозначно при $1 / f_{I}^{\prime}, f_{S}^{\prime} / f_{I}^{\prime} \in \mathbf{Z}$. Отображение фазовых пространств взаимно однозначно при $f_{I}^{\prime}= \pm 1, f_{S}^{\prime}=k \in \mathbf{Z}$, т.е. при $\mu=k S \pm I$. Условие Редже выполняется во всем фазовом пространстве только при $\mu=k S+I, k \in \mathbf{N}$.

Области другого вида невозможно взаимно однозначно отобразить в $\mathcal{F}$, если требовать, чтобы $\mu$ зависел только от $I$ и $S$. Мы также требуем, чтобы в спиновой части отображение было тождественным (спин ротатора равен спину струны). Эти требования сужают класс рассматриваемых отображений. Заметим в связи с этим, что при сужении канонических преобразований осциллятора (произвольных сохраняющих площадь диффеоморфизмов фазовой плоскости) на класс $\tilde{I}=f(I)(\tilde{I}$ не зависит от $\tau)$ учет сохранения глобальной топологии фазовой плоскости оставляет только преобразования вида $\tilde{I}=I, \tilde{\tau}=\tau+F(I)$. Аналогичное явление происходит в рассматриваемой задаче. Локально канонические преобразования фазового пространства существуют в классе $\mu=f(I, S) \forall f$. Требование сохранения глобальной топологии отбирает в этом классе дискретный набор преобразований.

Покажем, что на верхней границе области Редже струна должна быть прямолинейной. В фазовом пространстве осциллятора множество $\{I=0, \tau \in[0,2 \pi]\}$ представляет одну точку. Поэтому $(0, \tau, \vec{S}, \vec{n}) \rightarrow(\mu=k S, \tau, \vec{S}, \vec{e}=R(-k \tau) \vec{n}) \forall \tau$ должно представлять одну конфигурацию. Эволюция и врашение действуют на такую конфигурацию одинаково. Это возможно только в случае прямолинейной ( $k$ раз сложенной) струны.

Внутри области Редже (при $I \neq 0$ ) струна не должна быть прямолинейной, поскольку в этом случае в $\mathcal{F}$ возникают лишние отождествления. По той же причине внутри области опорная кривая не должна быть кратной и не должна обладать осевой симметрией, поскольку в этих случаях в $\mathcal{F}$ сушествуют дискретные преобразования, сохраняющие конфигурацию. Однако в частном случае, когда все опорные кривые в $\mathcal{M}_{2}$ являются кратными и/или осесимметричными, т.е. когда эти симметрии имеются не при исключительных значениях $\mu$ и $S$, а во всем фазовом пространстве, такое семейство можно взаимно однозначно отобразить в $\mathcal{F}$.

Осевая симметрия. Пусть все конфигурации (может быть, за исключением граничных) являются $d$-кратными и обладают осевой симметрией порядка $p$. Осевая симметрия разбивает опорную кривую на $p d$ симметричных участков. Сдвиг $T: \tau \rightarrow \tau+$

\footnotetext{
${ }^{2)}$ Для получения этих соотношений необходимо обратить коэффициентную матрицу симплектической формы и вычислить скобки Дирака на поверхности связей $\vec{n}^{2}=1, \vec{S} \vec{n}=0$.
} 
$2 \pi / p d$ эквивалентен повороту $\vec{Q}(\sigma)$ вокруг $\vec{S}$ на угол $\alpha=2 \pi \nu / p d$, где $\nu$ - число зацепления $\vec{Q}(\sigma)$ за ось симметрии $\vec{S}$ (см. рис. 4). Запишем $\alpha=2 \pi r / p, r=(\nu / d) \bmod p \in \mathbf{Z}_{p}$. При $p \neq 1 \quad 1 \leq r<p, r$ и $p$ взаимно просты (в противном случае $r=n \tilde{r}, p=n \tilde{p}$, $\tilde{p} \Delta \tau=2 \pi / n d, \quad \tilde{p} \alpha=2 \pi \tilde{r}$, т.е. опорная кривая является $n d$-кратной). При $p=1$ (нет осевой симметрии) $r=0$. Для опорных кривых, пересекаюших ось симметрии, $\nu$ не определено.Однако при непрерывной деформации кривой, сохраняющей осевую симметрию, $p d$ симметричных участков одновременно пересекают ось симметрии. При этом $\nu$ меняется на $p d$, а $r$ не меняется. Таким образом, в классе осесимметричных кривых $r$ является топологическим инвариантом, все кривые в $\mathcal{M}_{2}$ обладают одним и тем же $r$. В частности, для граничной $k$-кратной окружности $\nu=k=d(r+p n), n \in \mathbf{Z}$.

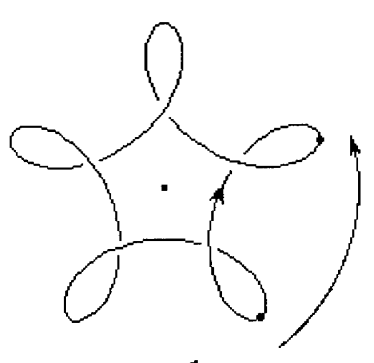

$\nu=1$

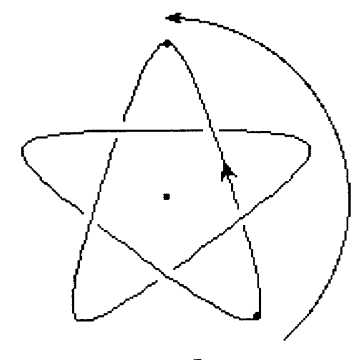

$\nu=2$

Рис. 4. Осесимметричные опорные кривые, $p=5, d=1$.

Конфигурация сохраняется при преобразовании

$$
U=T(2 \pi / p d) R(-2 \pi r / p)
$$

На верхней гранище области Редже могут происходить дополнительные вырождения, $k$ может не совпадать с $d$. Для $k$-кратной окружности дискретная симметрия $U$ является частным случаем непрерывной симметрии этой конфигурации: $T(C) R(-k C)$.

Вектор $\vec{n}=R(k \tau) \vec{e}$ сохраняется при преобразовании $U: \vec{n} \rightarrow R(2 \pi(k-r d) / p d) \vec{n}=\vec{n}$. Таким образом, в $\mathcal{F}$ преобразование $U$ является сдвигом $\tau \rightarrow \tau+2 \pi / p d$. Перейдем к новым каноническим переменным $\tau=\tilde{\tau} / p d, I=\tilde{I} \cdot p d$. В новых переменных $U: \tilde{\tau} \rightarrow \tilde{\tau}+2 \pi$, поэтому в новом фазовом пространстве нет избыточных отождествлений, оно находится во взаимно однозначном соответствии с $\mathcal{M}_{2}$. В следующем разделе будет показано, что при переходе к переменным $(\tilde{I}, \tilde{\tau})$ изменяется спин-массовый спектр системы.

$P$-отражение. Для плоских опорных кривых $\left(Q_{3}=0\right) P$-отражение является поворотом $R(\vec{S}, \pi)$. Для неплоских кривых $P$-отражение действует в $\mathcal{M}_{2}$ только при специальном выборе опорных кривых.

Поскольку $P \vec{Q}$ обладает теми же $\mu$ и $\vec{S}$, что и $\vec{Q}$, эти кривые имеют одинаковую форму и их можно совместить врашением вокруг $\vec{S}$ на некоторый угол. Таким образом, $P$ действует в $\mathcal{M}_{2}$ только в том случае, если опорные кривые обладают зеркально-поворотной симметрией $S_{2 p}$. Эта симметрия разбивает опорную кривую на 
$2 p d$ симметричных участков. Сдвиг $T(\Delta \tau), \Delta \tau=\pi / p d$, для такой кривой эквивалентен отражению П относительно плоскости, ортогональной $\vec{S}$, и повороту вокруг $\vec{S}$ на угол $\alpha=\pi \nu / p d$. (Плоские опорные кривые включаются сюда как частный случай $\Delta \tau=\alpha=0$.) При непрерывной деформации кривой $\nu / d$ может измениться на $2 p n$, при этом $r_{2}=(\nu / d) \bmod 2 p$ не меняется и $\alpha=\pi r_{2} / p$. Для граничной $k$-кратной окружности $\nu=k=d\left(r_{2}+2 p n\right)$. Заметим, что $\left(S_{2 p}\right)^{2}=C_{p}, S_{2 p}$-симметричная кривая при $p \neq 1$ является также $C_{p}$-симметричной. При $p=1$ кривая является $P$-симметричной для нечетных $\nu / d\left(r_{2}=1\right)$ и П-симметричной для четных $\nu / d\left(r_{2}=0\right)$, см. рис. 5 .

Преобразование П действует на данные конфигурации как $R(-\alpha) T(\Delta \tau)$, поэтому $P=\Pi R(\pi)=R(\pi-\alpha) T(\Delta \tau)$. Для вектора $\vec{n}=R(k \tau) \vec{e}: P \vec{n}=R\left(\pi+\pi\left(k-r_{2} d\right) / p d\right) \vec{n}=$ $R(\pi) \vec{n}$. Таким образом, в $\mathcal{F}$ для $S_{2 p}$-симметричных конфигураций $P=R(\pi) T(\pi / p d)$.

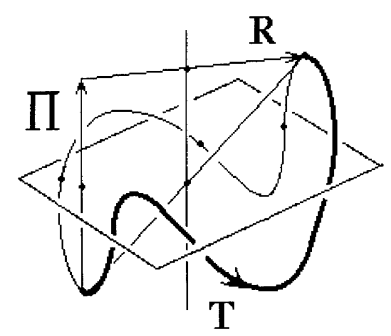

a)

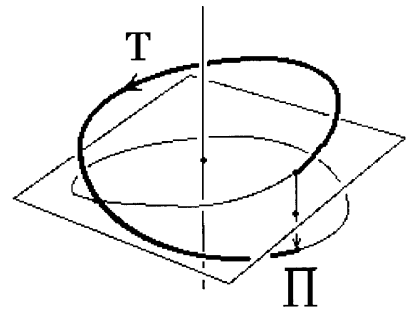

b)

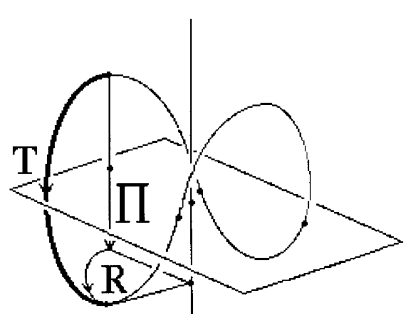

c)

Рис. 5. Опорные кривые с зеркально-поворотной симметрией, $a: p=1, \nu=1, r_{2}=1$; $b: p=1, \nu=2, r_{2}=0 ; c: p=2, \nu=1, r_{2}=1(d=1)$.

ЗАмечаниЕ. Точки фазового пространства $S=0$ являются особыми. В разделе 1 мы выбрали $\vec{e}_{3}=\vec{S} / S$. Кроме того, при канонических отображениях производились врашения векторов вокруг направления $\vec{S}$, гамильтониан также генерирует такие врашения. Эти вращения определены только при $S \neq 0$. В квантовой механике эту особенность можно устранить при соответствующем выборе квантовых аналогов $S$ и $\vec{n}_{i}$.

\section{4. КВАНТОВАНИЕ}

В предыдущем разделе мы рассмотрели 6-мерные подмногообразия фазового пространства струны, изоморфные фазовому пространству системы "осциллятор+ротатор". Воспользуемся квантовой теорией этой системы для квантования исследуемых многообразий.

Пространство состояний: прямое произведение фоковского пространства $\alpha|0\rangle=0$, $|I\rangle=\left(\alpha^{+}\right)^{I}|0\rangle,\left[\alpha, \alpha^{+}\right]=1$ ( $\alpha$ - квантовый аналог $\left.\sqrt{I} e^{-i \tau}\right)$ на пространство (квадратично интегрируемых) функций от вектора $\vec{n}$ на единичной сфере. При этом $\vec{S}=-i \vec{n} \times$ $\partial / \partial \vec{n}$. Выполнены соотношения

$$
\vec{S} \vec{n}=0, \quad\left[S^{i}, S^{j}\right]=i \epsilon_{i j k} S^{k}, \quad\left[S^{i}, n^{j}\right]=i \epsilon_{i j k} n^{k} .
$$




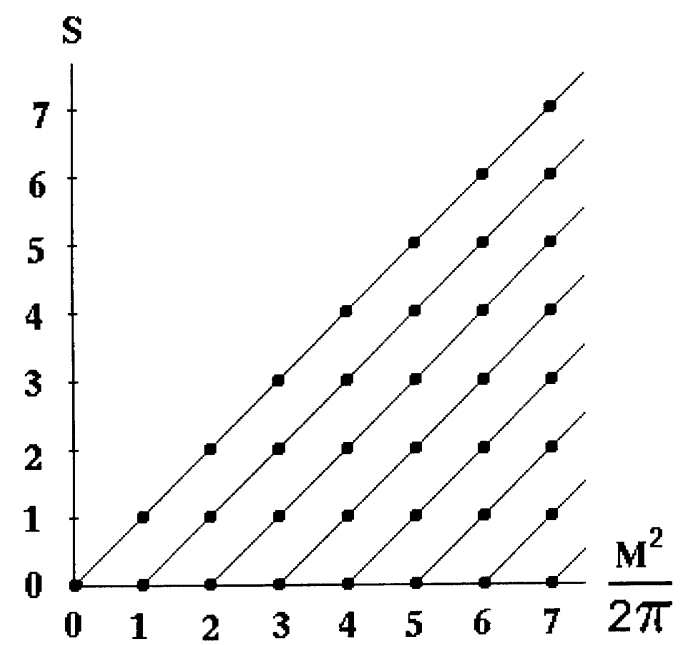

Рис. 6. Спектр масс для конфигураций типа "осциллятор+ротатор", $k=1$.

Базис пространства состояний: $\left|I S S^{3}\right\rangle=|I\rangle \cdot Y_{S S^{3}}(\vec{n})$.

Оператор квадрата массы: $\mu=k S+I+c_{0}, I=\alpha^{+} \alpha, c_{0}$-произвольная $c$-числовая константа. Оператор $S$ определяется соотношением [4] $S=\sqrt{\vec{S}^{2}+1 / 4}-1 / 2$, так что $\vec{S}^{2}=S(S+1)$. Оператор $S$ генерирует врашения $\vec{n}$ вокруг $\vec{S}$. В теории ротатора собственные значения $S=0,1,2 \ldots$ Спин-массовый спектр приведен на рис. 6 . Спектр состоит из линейных реджевских траекторий, $I$ является номером траектории. Состояния спектра невырожденны (без учета $(2 S+1)$-кратного вырождения по $\left.S^{3}\right)$.

Осевая симметрия. При квантовании $C_{p}$-симметричных конфигураций получается спектр $\mu=k S+p d \tilde{I}+c_{0}, \tilde{I}=0,1,2, \ldots$, т.е. в спектре рис. 6 необходимо оставить траектории с номерами, кратными $p d .^{3)}$

Этот результат можно получить также с помощью правила отбора по квантовому числу $U$. Преобразование $U=T(2 \pi / p d) \Leftrightarrow \alpha^{+} \rightarrow e^{i 2 \pi / p d} \alpha^{+}$можно реализовать с помошью унитарного оператора $U=e^{i 2 \pi I / p d}$. Для $U$-симметричных конфигураций $[\vec{Q}(\sigma), U]=0, \vec{Q}(\sigma)$ сохраняет $U$. Таким образом, пространство состояний разбивается на $p d$ изолированных секторов с разными $U$ : не существует операторов, построенных из конфигурации $\vec{Q}(\sigma)$, которые переводили бы друг в друга состояния из разных секторов. Спектры масс для этих секторов (рис. 7) отличаются друг от друга на сдвиг вдоль оси $\mu$. Поскольку этот сдвиг также можно получить изменением константы $c_{0}$, имеет смысл рассматривать только один сектор с $U=1$.

$P$-отражение. Для плоских конфигураций $P=e^{i \pi S}=(-1)^{S}$. Для $S_{2 p}$-симметричных конфигураций $P=e^{i \pi S+i \pi I / p d}=(-1)^{S+I / p d}=(-1)^{S+\tilde{I}}$.

\footnotetext{
${ }^{3)}$ Полученный спектр содержится в спектре для произвольных осесимметричных конфигураций, приведенном в работе [2].
} 


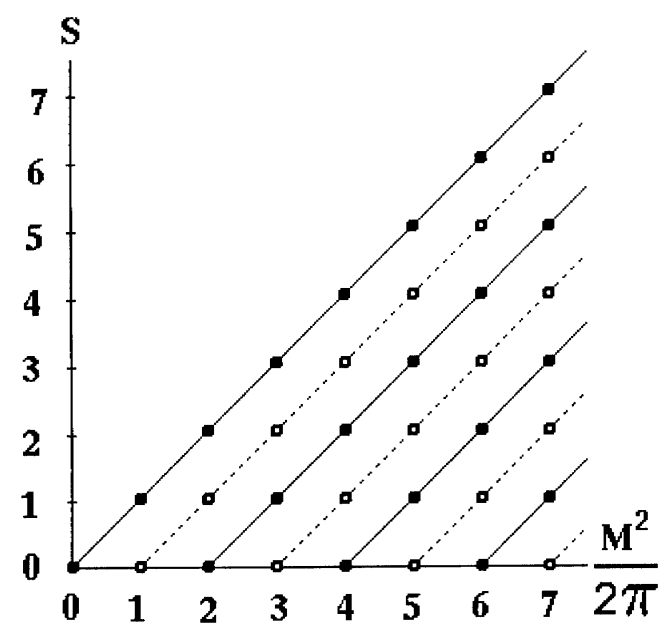

Рис. 7. Спектр масс для осесимметричной конфигурации с $p=2, d=1, k=1$. Пространство состояний разбито на 2 сектора с определенными значениями $U=(-1)^{I}$ : $U=+1$ (сплошные линии), $U=-1$ (штриховые линии).

ЗАмЕчАниЕ. В работе [5] построен оператор конфигурации $\vec{Q}(\sigma)$, удовлетворяющий всем необходимым свойствам: он эрмитов и правильно преобразуется при врашениях и эволюции. В [5] также проведено квантование 6-мерных многообразий другого типа многообразий, глобально изоморфных фазовому пространству волчка $\mathbf{R}^{3} \times S O(3)$.

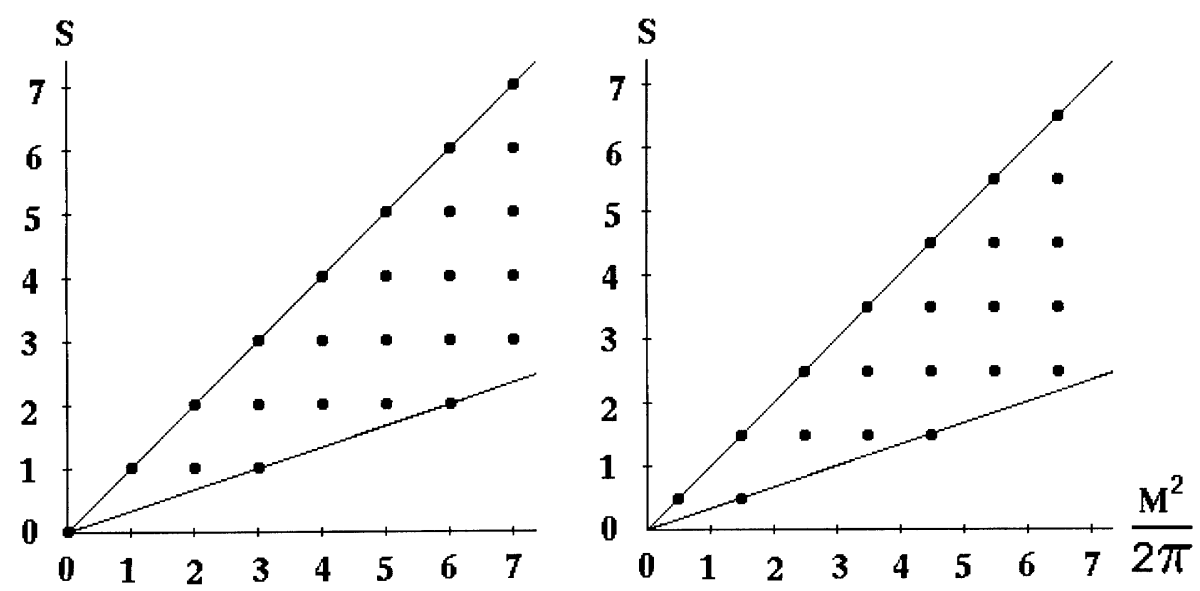

Рис. 8. Спектр масс для конфигураций типа "волчок".

Поскольку фундаментальная группа этого многообразия нетривиальна: $\pi_{1}(S O(3))=$ $\mathbf{Z}_{2}$, в квантовой теории возможны два представления $S O(3)$ : однозначное и двузначное, 
соответствуюшие целым и полуцелым значениям спина (рис. 8).

\section{ЗАКЛЮЧЕНИЕ}

Сформулируем полученные в работе результаты.

Показано, что 6-мерные врашательно и эволюционно-инвариантные подмногообразия фазового пространства струны при определенных ограничениях топологического характера на их вид допускают взаимно однозначное каноническое отображение на фазовое пространство системы "осциллятор+ротатор" и последующее квантование.

В построенной гамильтоновой теории спин струны является независимой канонической переменной, что обеспечивает явную лоренц-ковариантность квантовой теории.

Получены спин-массовые спектры состояний, и исследованы дискретные симметрии системы. Спектры состоят из бесконечного набора линейных реджевских траекторий. Интерсепт траекторий не фиксируется при квантовании и может быть выбран таким, чтобы в теории отсутствовал тахион.

Автор благодарит Э. А. Петросяна, Г. П. Пронько, А. В. Разумова и Л. Д. Соловьева за полезные обсуждения.

Работа вьполнена при финансовой поддержке Международной соросовской программы.

\section{Список литературы}

[1] G. P. Pron'ko. Rev. Math. Phys. 1990. V. 2. № 3. Р. 355; Г. П. Пронько. ТМФ. 1984. Т. 59. № 2. C. 240

[2] И. Н. Никитин. ЯФ. 1993. Т. 56. № 9. С. 230.

[3] Г. П. Пронько, А. В. Разумов. ТМФ. 1983. Т. 56. № 2. С. 192; В.И. Бородулин и др. ТМФ. 1985. Т. 65. № 1. С. 119; Е. Б. Бердников, Г. П. Пронько. ЯФ. 1991. Т. 54. № 3. C. 763 .

[4] Е. Б. Бердников, Г. Г. Нанобашвили, Г. П. Пронько. ЯФ. 1992. Т. 55. № 1. С. 203.

[5] И. Н. Никитин. Частные классы движений струны, квантуемые без аномалий: Препринт ИФВЭ 96-3. Протвино: ИФВЭ, 1996.

Поступила в редакцию 25.I.1996 г.

\section{N. Nikitin \\ SPECIAL TYPES OF STRING MOTIONS WITH ANOMALY-FREE QUANTIZATION}

In a phase space of the open bosonic string finite dimensional submanifolds are studied, whose quantization is free of anomalies at $d=4$. 